\section{Diferenciais geográficos, socioeconômicos e demográficos da qualidade da informação da causa básica de morte dos idosos no Brasil}

\author{
Geographic, demographic, and socioeconomic \\ differences in quality of data on cause of \\ death in Brazilian elders
}

\author{
1 Escola Nacional de Saúde \\ Pública Sergio Arouca, \\ Fundação Oswaldo Cruz, \\ Rio de Janeiro, Brasil. \\ 2 Instituto de Comunicação \\ e Informação Científica \\ e Tecnológica em Saúde, \\ Fundação Oswaldo Cruz, \\ Rio de Janeiro, Brasil. \\ ${ }^{3}$ Faculdade de Medicina, \\ Universidade Federal de \\ Minas Gerais, Belo Horizonte, \\ Brasil. \\ Correspondência \\ S. Kanso \\ Escola Nacional de Saúde \\ Pública Sergio Arouca, \\ Fundação Oswaldo Cruz. \\ Rua Leopoldo Bulhões 1480, \\ Rio de Janeiro, $R J$ \\ 21041-210, Brasil. \\ solange.kanso@gmail.com
}

\section{Abstract}

This study identifies geographic, demographic, and socioeconomic differences in the quality of data on underlying causes of death in the Brazilian elderly population. We used three quality indicators: the proportion of ill-defined causes, the proportion of unspecified causes, and the two proportions combined. We analyzed the main ill-defined and unspecified causes and the association between these indicators and individual characteristics on death certificates (DC) and characteristics of the municipalities. Unspecified causes exceed ill-defined causes, and both increase with age. Schooling, race, size of $m u$ nicipality, and per capita GDP were associated with quality of data (especially with ill-defined as compared to unspecified causes). Having received medical care decreased the odds of cause of death being recorded as ill-defined and increased the odds of unspecified records. Programs to improve quality of information on death certificates should focus on ill-defined and unspecified causes in the elderly.

Death Certificates; Underlying Cause of Death; Aged
Solange Kanso ${ }^{1}$

Dalia Elena Romero 2

Iúri da Costa Leite 1

Edgar Nunes de Moraes ${ }^{3}$

A Declaração de Óbito (DO), documento de caráter contínuo, permanente e obrigatório, padronizado em todo o território nacional - contém variáveis relevantes 1 e constitui fonte de informação fundamental, sendo que sua utilização está condicionada à qualidade dos dados 2,3 . No Brasil, apesar da expressiva melhora na coleta e qualidade dos dados de mortalidade 4 , ainda persistem problemas, destacando-se o sub-registro de óbitos e as falhas no preenchimento das informações, principalmente aquela referente à causa básica de morte 5,6,7.

A proporção de mortes por causas mal definidas, correspondentes ao capítulo XVIII da Classificação Internacional de Doenças - 10a Revisão (CID-10) 8 Sintomas, Sinais e Achados Anormais de Exames Clínicos e de Laboratório não Classificados em Outra Parte, tem sido amplamente utilizada e reconhecida como indicador para avaliar a qualidade da informação das causas de mortalidade 3,7,9,10,11. Em muitos casos, essa proporção é resultado da falta de infraestrutura assistencial, condições para o diagnóstico de doenças e, ainda, capacitação profissional para preenchimento das DO 12. Um percentual elevado de óbitos por causas mal definidas prejudica a compreensão do padrão, nível e causalidade da mortalidade 4,7 . No Brasil, análises da situação de saúde dos idosos ficam comprometidas pela elevada propor- 
ção de causas mal definidas nesta faixa etária 13, afetando, assim, o estudo geral da mortalidade no país, já que aproximadamente $60 \%$ do total de óbitos ocorridos anualmente correspondem a pessoas de 60 anos ou mais 7 .

Outro problema referente à qualidade da informação é a proporção de causas de óbitos inespecíficas, pois, embora sejam classificadas num grupo geral de causas, como a "doença não especificada do coração", não se conhece sua particularidade dentro da CID. Mathers et al. 3 elencaram um conjunto de causas inespecíficas, denominadas de códigos-lixo por Murray \& Lopez 14 , relativas às doenças cardiovasculares, às neoplasias e às causas externas, para classificar a qualidade das informações sobre a causa de óbito, em vários países.

Pouco se conhece sobre a participação das causas inespecíficas na estrutura da mortalidade no país, principalmente nesse segmento populacional. Em um estudo sobre uma coorte de idosos residentes em Bambuí, município de Minas Gerais, Brasil 11, por exemplo, observou-se que, enquanto a proporção de mortes por causas mal definidas diminuiu $77 \%$, passando de $14,3 \%$, em 1997-1999, para 3,3\%, em 2003-2005, a proporção de óbitos por causas inespecíficas permaneceu relativamente constante (em torno de $10 \%$ durante todo o período). A despeito da importância desse estudo, análises sobre a qualidade da informação devem ser realizadas em contextos mais amplos e variados.

A associação entre qualidade da informação sobre mortalidade e o nível de desenvolvimento socioeconômico das regiões do Brasil tem sido observada em vários estudos, principalmente para crianças menores de um ano 5,15,16. No entanto, são escassas as análises dessa natureza voltadas para a população idosa 13 .

Assim, o objetivo principal deste trabalho é identificar diferenciais geográficos e desigualdades socioeconômicas e demográficas no que diz respeito ao padrão de qualidade da informação sobre a causa básica de morte entre os idosos, no Brasil. Para tanto, foram utilizados os dados referentes às causas mal definidas e às inespecíficas, para 2007. Além disso, procura-se associar a qualidade dessa informação à completitude do preenchimento de outras variáveis da DO.

\section{Material e métodos}

A principal fonte para obtenção dos microdados de óbitos de idosos, definidos como indivíduos com 60 anos ou mais, foi o Sistema de Informações sobre Mortalidade (SIM), do Departamento de Informática do SUS (DATASUS) do Ministério da Saúde, referente a 2007. Informações sobre as populações dos municípios e seus respectivos Produto Interno Bruto (PIB) per capita foram obtidas do Instituto Brasileiro de Geografia e Estatística (IBGE), para o mesmo ano. As análises foram realizadas de acordo com os seguintes níveis de agregação: individual, área geográfica de residência segundo as Regiões, Unidades da Federação (UF), capitais e municípios que não pertencem à capital. Para análise dos dados empregou-se o programa estatístico SPSS 15.0 para Windows (SPSS Inc., Chicago, Estados Unidos).

Utilizou-se a CID-10 8 em níveis de três a quatro caracteres dos códigos da causa básica de morte.

A análise foi feita por meio de três indicadores: proporção de causas mal definidas; proporção de causas inespecíficas; e combinação da proporção de causas mal definidas e proporção de causas inespecíficas.

\section{Indicadores de qualidade da causa básica de morte}

- Proporção de causas mal definidas: pertencem ao capítulo XVIII da CID-10 (Sintomas, Sinais e Achados Anormais de Exames Clínicos e de Laboratório não Classificados em Outra Partecódigo R00 a R99).

PCMD = ((óbitos do capítulo XVIII) / (total de óbitos) $) * 100$

- Proporção de causas inespecíficas: correspondem às causas de mortalidade não pertencentes ao capítulo XVIII, consideradas imprecisas ou não suficientemente definidas. Analisou-se a pertinência, para idosos, das categorias de códigos-lixo definidas por Mathers et al. 3 (Doenças Cardiovasculares, Cânceres e Causas Externas) e incluíram-se causas inespecíficas dos nove capítulos da CID mais incidentes entre os idosos, definindo-se assim a seguinte lista: (a) doenças do aparelho respiratório (capítulo IX): I46, I49.0, I49.9, I50, I51.5, I51.6, I51.8, I51.9, I52, I67.8, I68.8, I69.8, I70.9, I97.8, I97.9, I98.8 e I99; (b) neoplasias (capítulo II): C76, C77, C79, C80 e C97; (c) doenças do aparelho respiratório (capítulo X): J06.8, J39.8, J39.9, J95.8, J95.9 e J98; (d) doenças endócrinas nutricionais e metabólicas (capítulo IV): E56.8, E56.9, E61.8, E61.9, E63.8, E63.9, E64.8, E64.9, E88.9, E89.9 e E90; (e) doenças do aparelho digestivo (capítulo XI): K92.0, K92.1, K92.2, K92.8, K92.9 e K93.8; (f) causas externas de morbidade e mortalidade (capítulo XX): Y10-Y34, Y85, Y86, Y87 e Y89.9; (g) algumas doenças infecciosas e parasitárias (capítulo I): A48.8, A49.8, B33.8, B34.8, B34.9, B89, B96.8, 
B97.8 e B99; (h) doenças do aparelho geniturinário (capítulo XIV): N99.8 e N99.9; (i) doenças do sistema nervoso (capítulo VI): G97.8, G97.9, G98 e G99.8.

PCI = ((óbitos de CI) / (total de óbitos - óbitos do capítulo XVIII) $) * 100$

Excluem-se do denominador os óbitos registrados como causas mal definidas, com o objetivo de dimensionar o peso das causas inespecíficas no total de óbitos definido pelas causas tradicionalmente consideradas "bem definidas".

- Combinação de proporção de causas mal definidas e proporção das causas inespecíficas: o terceiro indicador foi criado por meio da combinação das proporções das causas mal definidas e inespecíficas.

\section{Critérios de análise e categorias da qualidade} da informação segundo indicadores

Para a proporção de causas mal definidas, investigaram-se as três principais causas de morte do capítulo XVIII da CID-10. A classificação da qualidade das informações pautou-se, inicialmente, no pressuposto de que proporção de causas mal definidas $<5 \%$ e proporção de causas mal definidas $\geq 15 \%$ estariam associadas, respectivamente, à alta e à baixa qualidade do registro 17,18,19. Categorias intermediárias foram criadas de modo a se obter uma distribuição mais uniforme dos grupos, resultando, assim, no seguinte critério: (a) proporção de causas mal definidas menor do que 5\% (<5\%): qualidade altamente adequada; (b) proporção de causas mal definidas entre 5\% e $7 \%$ ( $\geq 5 \%$ e $<7 \%$ ): qualidade adequada; (c) proporção de causas mal definidas entre $7 \%$ e $10 \%$ ( $\geq 7 \%$ e < 10\%): qualidade pouco adequada; (d) proporção de causas mal definidas entre $10 \%$ e $15 \%$ ( $\geq 10 \%$ e < 15\%): qualidade inadequada; (e) proporção de causas mal definidas igual ou maior do que $15 \%$ ( $\geq 15 \%$ ): qualidade altamente inadequada.

$\mathrm{Na}$ análise referente à proporção de causas inespecíficas também foram investigadas as três principais causas no total das inespecíficas. Uma vez que a variabilidade na proporção de causas inespecíficas foi bem menor do que aquela observada para as mal definidas, utilizou-se, para classificação da qualidade da informação relativa à proporção de causas inespecíficas, apenas o valor mediano da distribuição $(10,8)$, isto é: (a) proporção de causas inespecíficas menor do que 10,8 \% (< 10,8\%): qualidade adequada; (b) proporção de causas inespecíficas maior ou igual a $10,8 \%$ ( $\geq 10,8 \%$ ): qualidade inadequada.
No que se refere ao indicador combinado, foi elaborada a seguinte classificação quanto à qualidade das informações: (a) proporção de causas mal definidas menor do que 5\% $(<5 \%)$ e proporção de causas inespecíficas menor do que 10,8\% (< 10,8\%): alta qualidade; (b) proporção de causas mal definidas menor do que $5 \%(<5 \%)$ e proporção de causas inespecíficas maior ou igual a $10,8 \%$ ( $\geq 10,8 \%$ ), ou proporção de mortes por causas mal definidas entre $5 \%$ e $7 \%(\geq 5 \%$ e $<7 \%)$ e proporção de causas inespecíficas menor do que 10,8\% (<10,8\%): qualidade aceitável; (c) proporção de causas mal definidas menor entre $5 \%$ e $7 \%$ ( $\geq 5 \%$ e $<7 \%$ ) e proporção de causas inespecíficas maior ou igual a 10,8\% ( $\geq 10,8 \%$ ), ou proporção de causas mal definidas entre $7 \%$ e $10 \%$ ( $\geq$ $7 \%$ e $<10 \%$ ) e proporção de causas inespecíficas menor do que $10,8 \%(<10,8 \%)$ : qualidade regular; (d) proporção de causas mal definidas entre $7 \%$ e $10 \%$ ( $\geq 7 \%$ e < $10 \%$ ) e proporção de causas inespecíficas maior ou igual a 10,8\% ( $\geq 10,8 \%$ ), ou proporção de causas mal definidas maior ou igual a $10 \%$ ( $\geq 10 \%)$ : qualidade inadequada.

\section{Análise da associação da qualidade da informação da causa de morte com fatores individuais e contextuais}

Tendo em vista que a qualidade da informação pode estar relacionada com o município de residência do idoso, para identificação de fatores associados à má qualidade do preenchimento da causa básica de morte entre idosos foram utilizados modelos de regressão multinomial hierárquico 20,21, em que a variável resposta é nominal com três categorias referentes ao registro da causa do óbito: mal definida, inespecífica e bem definida.

As variáveis explicativas foram subdivididas em dois níveis: individual, representado pelos idosos; e contextual, que corresponde aos municípios. As variáveis individuais contidas na DO e consideradas nos modelos foram agrupadas em três blocos: demográficas, que compreendem sexo e grupos de idade (60-69, 70-79, 80-89, 90-99 e 100 anos ou mais); socioeconômicas, que englobam raça/cor (branca, preta, amarela, parda, indígena e ignorada) e anos de estudo (nenhum, 1-3, 4-7, 8 anos ou mais e ignorado); e atenção à morte, abrangendo assistência médica (sim, não e ignorada) e local de ocorrência do óbito (estabelecimentos de saúde, domicílio, outros e ignorado). Já as variáveis contextuais foram agrupadas na categoria desenvolvimento, compreendendo a população do município (menos de 20 mil habitantes, 20-50 mil, mais de 50-100 mil, mais de 100-500 mil e mais de 500 mil), se o município é capital ou não, e pobreza, expressa 
pelo PIB per capita e incluída no modelo como variável contínua.

A associação entre a qualidade da informação sobre a causa básica de mortalidade dos idosos e a completitude de outras variáveis da DO foi testada nos modelos, comparando-se a categoria "ignorada" com a categoria de referência de algumas variáveis individuais.

Os parâmetros dos modelos multinomiais hierárquicos não ajustados foram obtidos utilizando-se como procedimento de estimação a Quase-Verossimilhança Penalizada (PQL) de segunda ordem, disponível no programa MLwiN, versão 2.10 (Centre for Multilevel Modelling, Bristol, Reino Unido), e foram interpretados como razões de chance.

\section{Resultados}

A Figura 1 mostra que a proporção de causas inespecíficas, com exceção das idades extremas, tem comportamento semelhante à proporção de causas mal definidas, mas em níveis mais elevados. As causas mal definidas apresentaram proporções ligeiramente maiores para os homens, enquanto as inespecíficas foram superiores entre as mulheres.
A qualidade da informação piora com o avanço da idade dos idosos para os dois indicadores comparados. No caso da proporção das inespecíficas, o aumento é relativamente constante, enquanto que para as causas mal definidas a piora da informação acentua-se a partir dos 85 anos, atingindo quase $22 \%$ dos óbitos de idosos com 100 anos ou mais de idade.

As Tabelas 1, 2 e 3 apresentam a proporção de causas mal definidas e suas principais causas, segundo regiões, UF, municípios capitais e demais municípios dos estados. No Brasil, 8,4\% das causas de óbito de idosos foram classificadas como mal definidas, oscilando acentuadamente entre as UF e regiões (1,6\% em Mato Grosso do Sul e $24,1 \%$ no Amapá; 4,7\% no Centro-oeste e 16,5\% no Norte). Observaram-se menores proporções de causas mal definidas nas capitais, com exceção do Município de Fortaleza, com o dobro da proporção de causas mal definidas observada nos demais municípios do estado.

Quanto às principais causas mal definidas do capítulo XVIII da CID-10, verificou-se concentração em morte sem assistência (38,5\%), de código R98, e causas mal definidas sem nenhuma especificação (35,2\%), as quais são denominadas de outras causas mal definidas e as não especificadas de mortalidade (R99). A terceira causa mais

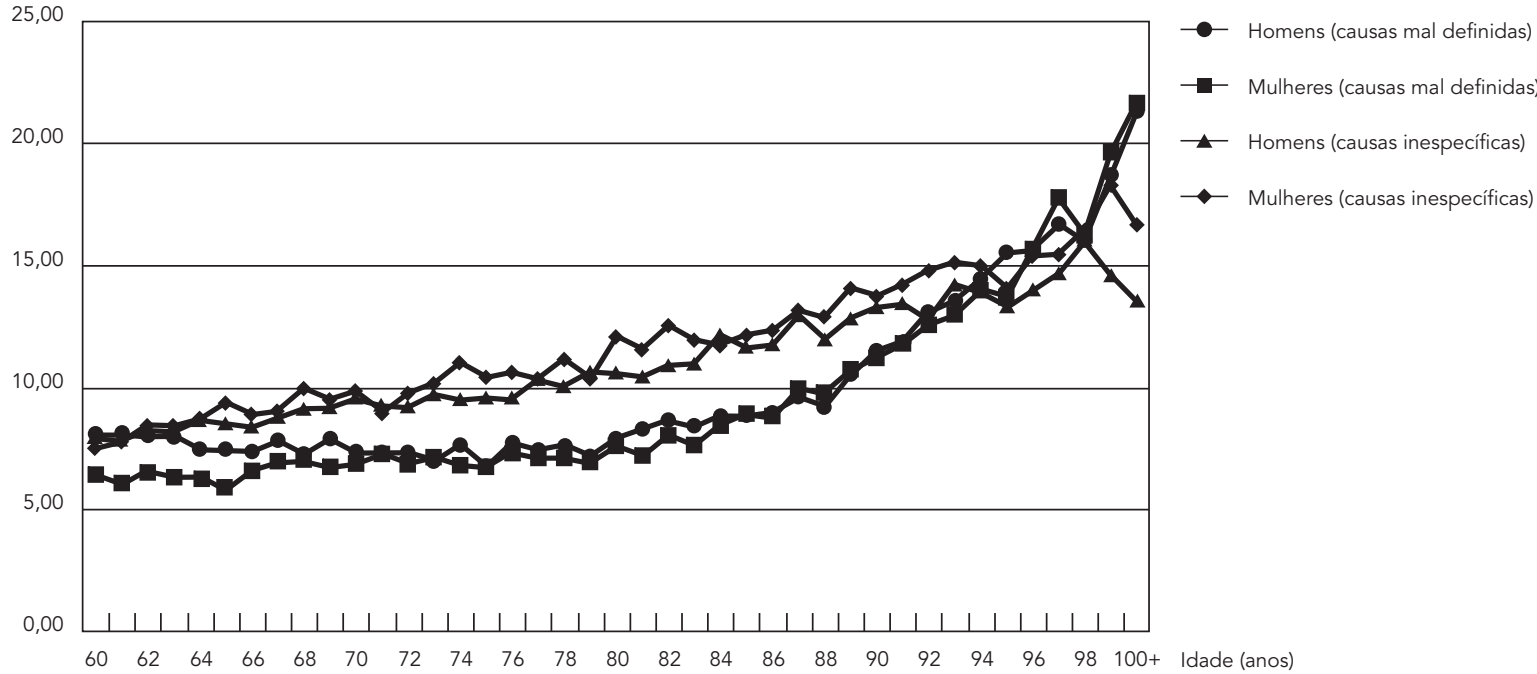

Fonte: Sistema de Informações sobre Mortalidade (SIM), Ministério da Saúde. 
Número e proporção de óbitos entre idosos por causas mal definidas e participação das três principais causas mal definidas, por Unidade da Federação (UF). Brasil, 2007.

\begin{tabular}{|c|c|c|c|c|c|}
\hline \multirow[t]{2}{*}{ Região/UF } & \multirow{2}{*}{$\begin{array}{l}\text { Total de } \\
\text { óbitos }\end{array}$} & \multirow{2}{*}{$\begin{array}{c}\% \text { de causas mal } \\
\text { definidas }\end{array}$} & \multicolumn{3}{|c|}{ Participação das causas no capítulo XVIII } \\
\hline & & & $\begin{array}{c}\text { Morte sem assistência } \\
\text { (R98) }\end{array}$ & $\begin{array}{l}\text { Parada respiratória } \\
\text { (R09.2) }\end{array}$ & $\begin{array}{c}\text { Outras causas mal definidas e as } \\
\text { não especificadas (R99) }\end{array}$ \\
\hline Norte & 4.402 & 16,5 & 51,3 & 8,5 & 26,6 \\
\hline $\mathrm{RO}$ & 263 & 10,0 & 11,8 & 12,2 & 22,8 \\
\hline $\mathrm{AC}$ & 103 & 7,8 & 53,4 & 12,6 & 23,3 \\
\hline AM & 1.271 & 23,0 & 56,9 & 3,7 & 30,9 \\
\hline RR & 33 & 5,7 & 27,3 & 12,1 & 21,2 \\
\hline PA & 2.454 & 18,8 & 57,6 & 9,4 & 21,8 \\
\hline$A P$ & 169 & 24,1 & 6,5 & 5,9 & 71,6 \\
\hline TO & 109 & 3,7 & 15,6 & 33,9 & 25,7 \\
\hline Nordeste & 14.913 & 9,8 & 61,8 & 9,3 & 16,1 \\
\hline MA & 1.238 & 10,0 & 48,6 & 20,3 & 10,0 \\
\hline $\mathrm{PI}$ & 464 & 5,2 & 43,3 & 10,6 & 9,1 \\
\hline CE & 1.527 & 6,2 & 33,1 & 22,9 & 28,7 \\
\hline RN & 333 & 3,7 & 16,2 & 12,3 & 35,4 \\
\hline PB & 1.331 & 9,6 & 49,3 & 18,4 & 15,9 \\
\hline PE & 2.020 & 6,5 & 68,7 & 5,8 & 12,7 \\
\hline$A L$ & 780 & 9,3 & 64,9 & 5,8 & 14,9 \\
\hline SE & 486 & 8,8 & 42,8 & 4,1 & 40,7 \\
\hline BA & 6.734 & 17,9 & 75,6 & 3,9 & 13,4 \\
\hline Sudeste & 25.083 & 8,2 & 26,2 & 8,5 & 48,1 \\
\hline MG & 7.626 & 11,3 & 41,3 & 8,2 & 34,3 \\
\hline ES & 382 & 3,6 & 57,1 & 7,1 & 14,7 \\
\hline RJ & 6.939 & 9,4 & 0,5 & 8,7 & 78,5 \\
\hline $\mathrm{SP}$ & 10.136 & 6,6 & 31,3 & 8,6 & 38,8 \\
\hline Sul & 6.497 & 6,0 & 29,0 & 11,7 & 34,9 \\
\hline PR & 1.979 & 5,2 & 36,9 & 12,3 & 17,0 \\
\hline SC & 1.993 & 10,1 & 40,1 & 10,2 & 36,8 \\
\hline RS & 2.525 & 5,0 & 13,9 & 12,4 & 47,5 \\
\hline Centro-oeste & 1.609 & 4,7 & 18,1 & 13,5 & 36,4 \\
\hline MS & 118 & 1,6 & 17,8 & 5,1 & 39,0 \\
\hline $\mathrm{MT}$ & 237 & 3,8 & 8,0 & 13,5 & 48,5 \\
\hline GO & 1.128 & 7,2 & 21,6 & 14,5 & 31,9 \\
\hline DF & 126 & 2,5 & 5,6 & 12,7 & 51,6 \\
\hline Brasil & 52.504 & 8,4 & 38,5 & 9,3 & 35,2 \\
\hline
\end{tabular}

AC: Acre; AL: Alagoas; AM: Amazonas; AP: Amapá; BA: Bahia; CE: Ceará; DF: Distrito Federal; ES: Espírito Santo; GO: Goiás; MA: Maranhão; MG: Minas Gerais; MS: Mato Grosso do Sul; MT: Mato Grosso; PA: Pará; PB: Paraíba; PE: Pernambuco; PI: Piauí; PR: Paraná; RJ: Rio de Janeiro; RN: Rio Grande do Norte; RS: Rio Grande do Sul; RO: Rondônia; RR: Roraima; SC: Santa Catarina; SE: Sergipe; SP: São Paulo; TO: Tocantins.

Fonte: Sistema de Informações sobre Mortalidade (SIM), Ministério da Saúde.

frequente foi a parada respiratória (R09.2), com $9,3 \%$. Tal estrutura de causas varia entre UF, regiões e tipo de município, mas, de modo geral, essas três representam aproximadamente $80 \%$ do total de causas mal definidas.

As causas classificadas como morte sem assistência foram, na maioria das UF, mais frequen- tes nos municípios que não são capitais. A Bahia foi a UF que registrou a maior proporção de morte sem assistência (75,6\%), sendo esta ocorrência muito baixa na capital Salvador (1,9\%). Já no Rio de Janeiro, a maior proporção correspondeu às outras causas mal definidas e as não especificadas $(78,5 \%)$, relevantes tanto na capital como 
Tabela 2

Número e proporção de óbitos entre idosos por causas mal definidas e participação das três principais causas mal definidas, por capitais. Brasil, 2007.

\begin{tabular}{|c|c|c|c|c|c|}
\hline \multirow[t]{2}{*}{ Região/UF } & \multirow{2}{*}{$\begin{array}{l}\text { Total de } \\
\text { óbitos }\end{array}$} & \multirow{2}{*}{$\begin{array}{c}\% \text { de causas mal } \\
\text { definidas }\end{array}$} & \multicolumn{3}{|c|}{ Participação das causas no capítulo XVIII } \\
\hline & & & $\begin{array}{c}\text { Morte sem assistência } \\
\text { (R98) }\end{array}$ & $\begin{array}{c}\text { Parada respiratória } \\
\text { (R09.2) }\end{array}$ & $\begin{array}{c}\text { Outras causas mal definidas e as não } \\
\text { especificadas (R99) }\end{array}$ \\
\hline Norte & 1.136 & 11,2 & 24,7 & 7,8 & 49,8 \\
\hline $\mathrm{RO}$ & 79 & 9,9 & 0,0 & 6,3 & 8,9 \\
\hline$A C$ & 34 & 4,7 & 67,6 & 20,6 & 8,8 \\
\hline AM & 592 & 16,8 & 41,0 & 1,4 & 47,0 \\
\hline RR & 22 & 5,2 & 40,9 & 4,5 & 13,6 \\
\hline PA & 303 & 7,6 & 1,0 & 20,8 & 66,0 \\
\hline AP & 102 & 21,4 & 2,9 & 2,9 & 73,5 \\
\hline TO & 4 & 1,8 & 0,0 & 50,0 & 0,0 \\
\hline Nordeste & 1.124 & 3,3 & 26,6 & 16,9 & 39,0 \\
\hline MA & 51 & 2,1 & 5,9 & 17,6 & 29,4 \\
\hline $\mathrm{PI}$ & 7 & 0,3 & 28,6 & 28,6 & 0,0 \\
\hline CE & 653 & 9,5 & 43,8 & 22,2 & 26,2 \\
\hline $\mathrm{RN}$ & 44 & 1,8 & 4,5 & 4,5 & 45,5 \\
\hline PB & 34 & 1,4 & 0,0 & 14,7 & 29,4 \\
\hline PE & 27 & 0,5 & 0,0 & 3,7 & 48,1 \\
\hline$A L$ & 48 & 1,8 & 2,1 & 14,6 & 20,8 \\
\hline SE & 51 & 3,2 & 2,0 & 2,0 & 76,5 \\
\hline $\mathrm{BA}$ & 209 & 2,9 & 1,9 & 8,6 & 76,6 \\
\hline Sudeste & 3.769 & 4,4 & 0,8 & 7,9 & 75,5 \\
\hline MG & 449 & 5,3 & 3,1 & 4,0 & 81,5 \\
\hline ES & 12 & 1,1 & 0,0 & 0,0 & 83,3 \\
\hline RJ & 2.961 & 8,7 & 0,0 & 8,5 & 76,7 \\
\hline SP & 347 & 0,8 & 4,6 & 7,5 & 56,8 \\
\hline Sul & 181 & 1,2 & 7,7 & 11,6 & 56,4 \\
\hline PR & 68 & 1,1 & 13,2 & 26,5 & 25,0 \\
\hline $\mathrm{SC}$ & 12 & 1,0 & 16,7 & 16,7 & 25,0 \\
\hline RS & 101 & 1,4 & 3,0 & 1,0 & 81,2 \\
\hline Centro-oeste & 279 & 2,2 & 2,9 & 9,3 & 47,3 \\
\hline MS & 20 & 0,8 & 0,0 & 10,0 & 25,0 \\
\hline MT & 32 & 2,4 & 3,1 & 12,5 & 43,8 \\
\hline GO & 101 & 2,6 & 0,0 & 4,0 & 47,5 \\
\hline DF & 126 & 2,5 & 5,6 & 12,7 & 51,6 \\
\hline Brasil & 6.489 & 4,1 & 9,8 & 9,6 & 62,9 \\
\hline
\end{tabular}

AC: Acre; AL: Alagoas; AM: Amazonas; AP: Amapá; BA: Bahia; CE: Ceará; DF: Distrito Federal; ES: Espírito Santo; GO: Goiás; MA: Maranhão; MG: Minas Gerais; MS: Mato Grosso do Sul; MT: Mato Grosso; PA: Pará; PB: Paraíba; PE: Pernambuco; PI: Piauí; PR: Paraná; RJ: Rio de Janeiro; RN: Rio Grande do Norte; RS: Rio Grande do Sul; RO: Rondônia; RR: Roraima; SC: Santa Catarina; SE: Sergipe; SP: São Paulo; TO: Tocantins; UF: Unidade da Federação.

Fonte: Sistema de Informações sobre Mortalidade (SIM), Ministério da Saúde.

nos demais municípios. A parada respiratória respondeu por mais de $20 \%$ do total das mal definidas em Tocantins, Maranhão e Ceará. Entre as capitais, valores elevados foram observados em Teresina (28,6\%) e Palmas (50\%).

Ainda em relação às causas mal definidas, a classificação da qualidade da informação confirmou as diferenças acentuadas entre as diversas áreas geográficas, especialmente entre municípios capitais e não-capitais. Tanto no total do Brasil quanto no conjunto dos municípios que não são capitais, as informações sobre as causas mal definidas apresentaram qualidade pouco adequada $(\geq 7 \%$ e $<10 \%$ de proporção de causas mal definidas). A Região Norte registrou qualidade altamente inadequada ( $\geq 15 \%$ de proporção 
Número e proporção de óbitos entre idosos por causas mal definidas e participação das três principais causas mal definidas, por municípios não-capitais. Brasil, 2007.

\begin{tabular}{|c|c|c|c|c|c|}
\hline \multirow[t]{2}{*}{ Região/UF } & \multirow{2}{*}{$\begin{array}{l}\text { Total de } \\
\text { óbitos }\end{array}$} & \multirow{2}{*}{$\begin{array}{c}\% \text { de causas mal } \\
\text { definidas }\end{array}$} & \multicolumn{3}{|c|}{ Participação das causas no capítulo XVIII } \\
\hline & & & $\begin{array}{c}\text { Morte sem assistência } \\
\text { (R98) }\end{array}$ & $\begin{array}{l}\text { Parada respiratória } \\
\text { (R09.2) }\end{array}$ & $\begin{array}{c}\text { Outras causas mal definidas e as não } \\
\text { especificadas (R99) }\end{array}$ \\
\hline Norte & 3.266 & 19,7 & 60,6 & 8,7 & 18,5 \\
\hline $\mathrm{RO}$ & 184 & 10,0 & 16,8 & 14,7 & 28,8 \\
\hline$A C$ & 69 & 11,3 & 46,4 & 8,7 & 30,4 \\
\hline AM & 679 & 34,1 & 70,7 & 5,7 & 16,9 \\
\hline RR & 11 & 7,1 & 0,0 & 27,3 & 36,4 \\
\hline PA & 2.151 & 23,7 & 65,6 & 7,8 & 15,6 \\
\hline$A P$ & 67 & 29,9 & 11,9 & 10,4 & 68,7 \\
\hline TO & 105 & 3,9 & 16,2 & 33,3 & 26,7 \\
\hline Nordeste & 13.789 & 11,7 & 64,7 & 8,6 & 14,3 \\
\hline MA & 1.187 & 12,0 & 50,5 & 20,4 & 9,2 \\
\hline $\mathrm{Pl}$ & 457 & 6,8 & 43,5 & 10,3 & 9,2 \\
\hline CE & 874 & 4,9 & 25,1 & 23,5 & 30,7 \\
\hline RN & 289 & 4,4 & 18,0 & 13,5 & 33,9 \\
\hline PB & 1.297 & 11,4 & 50,6 & 18,5 & 15,5 \\
\hline PE & 1.993 & 7,9 & 69,6 & 5,9 & 12,2 \\
\hline $\mathrm{AL}$ & 732 & 12,7 & 69,0 & 5,2 & 14,5 \\
\hline SE & 435 & 11,1 & 47,6 & 4,4 & 36,6 \\
\hline BA & 6.525 & 21,5 & 78,0 & 3,7 & 11,4 \\
\hline Sudeste & 21.314 & 9,6 & 30,7 & 8,6 & 43,2 \\
\hline MG & 7.177 & 12,1 & 43,7 & 8,5 & 31,3 \\
\hline ES & 370 & 3,8 & 58,9 & 7,3 & 12,4 \\
\hline RJ & 3.978 & 9,9 & 0,8 & 8,9 & 79,9 \\
\hline $\mathrm{SP}$ & 9.789 & 8,7 & 32,3 & 8,6 & 38,2 \\
\hline Sul & 6.316 & 6,7 & 29,6 & 11,7 & 34,3 \\
\hline PR & 1.911 & 6,0 & 37,8 & 11,8 & 16,7 \\
\hline SC & 1.981 & 10,7 & 40,3 & 10,1 & 36,9 \\
\hline RS & 2.424 & 5,6 & 14,4 & 12,9 & 46,1 \\
\hline Centro-oeste & 1.330 & 6,1 & 21,3 & 14,4 & 34,1 \\
\hline MS & 98 & 1,9 & 21,4 & 4,1 & 41,8 \\
\hline MT & 205 & 4,1 & 8,8 & 13,7 & 49,3 \\
\hline GO & 1.027 & 8,7 & 23,8 & 15,5 & 30,4 \\
\hline DF & 0 & 0,0 & 0,0 & 0,0 & 0,0 \\
\hline Brasil & 46.015 & 9,8 & 42,6 & 9,2 & 31,3 \\
\hline
\end{tabular}

AC: Acre; AL: Alagoas; AM: Amazonas; AP: Amapá; BA: Bahia; CE: Ceará; DF: Distrito Federal; ES: Espírito Santo; GO: Goiás; MA: Maranhão; MG: Minas Gerais; MS: Mato Grosso do Sul; MT: Mato Grosso; PA: Pará; PB: Paraíba; PE: Pernambuco; PI: Piauí; PR: Paraná; RJ: Rio de Janeiro; RN: Rio Grande do Norte; RS: Rio Grande do Sul; RO: Rondônia; RR: Roraima; SC: Santa Catarina; SE: Sergipe; SP: São Paulo; TO: Tocantins; UF: Unidade da Federação.

Fonte: Sistema de Informações sobre Mortalidade (SIM), Ministério da Saúde.

de causas mal definidas), sendo exceções, nesta região, o Estado de Tocantins, com proporção bem pequena de causas mal definidas $(3,7 \%)$, as capitais Rio Branco (Acre) e Palmas (Tocantins) e os demais municípios de Tocantins. Já no Centro-oeste, a qualidade foi classificada como altamente adequada (proporção de causas mal definidas < 5\%), com exceção de Goiás, com a classificação pouco adequada (entre 7\% e 10\% de proporção de causas mal definidas). Destaca-se que, em Goiânia (Goiás), a qualidade da informação foi altamente adequada. Para o total das capitais do país, as informações apresentaram qualidade altamente adequada, com exceção da 
Região Norte, em que foram classificadas como inadequadas.

Considerando-se a proporção de causas inespecíficas para o Brasil (10,6\%), a qualidade das informações sobre causas básicas de morte de idosos foi classificada como adequada (Tabelas 4, 5 e 6). Diferenças regionais também foram observadas na proporção de causas inespecíficas, embora menos acentuadas do que aquelas referentes às causas mal definidas. Nas UF, a proporção de causas inespecíficas variou entre 8,4\%, no Espírito Santo, e 13,7\%, na Bahia. Entre as capitais, Vitória (Espírito Santo) registrou a menor proporção (5,3\%), enquanto Teresina (Piauí) apresentou a maior (15,6\%). Já em relação aos municípios não-capitais, a menor proporção foi verificada em Roraima $(7,6 \%)$ e a maior no Acre (14,1\%). De modo geral, o conjunto dos municípios não-capitais registrou proporção de inespecíficas maior, quando comparado ao total das capitais. Esse padrão não foi observado no Nordeste, com exceção de São Luís (Maranhão), Natal (Rio Grande do Norte) e João Pessoa (Paraíba). Na Região Norte, a única exceção foi a capital Boa Vista (Roraima).

As três principais causas de morte inespecíficas responderam por mais da metade dos óbitos, com predominância de insuficiência cardíaca congestiva (I50.0), seguida por outras doenças cerebrovasculares especificadas (I67.8) e insuficiência cardíaca não especificada (I50.9). A maior proporção de óbitos devido à insuficiência cardíaca congestiva foi observada no Piauí $(31,8 \%)$, enquanto a menor foi registrada no Rio Grande do Sul (13,3\%). As outras doenças cerebrovasculares especificadas tiveram maior incidência nas capitais do Nordeste e no Centro-oeste, com destaque para Teresina (38,9\%) e Campo Grande (Mato Grosso do Sul - 29,3\%). Nos municípios não-capitais, a maior proporção foi encontrada em Roraima $(36,4 \%)$ e a menor no Rio Grande do Sul (12,6\%).

Considerando-se o indicador combinado (proporção de causas mal definidas e proporção causas inespecíficas), observou-se que grande parte das UF, principalmente os municípios nãocapitais das regiões Norte e Nordeste, apresentou qualidade regular ou inadequada da informação. As capitais registraram qualidade alta e aceitável, com exceção de Belém (Pará), Porto Velho (Rondônia), Manaus (Amazonas), Macapá (Amapá), Fortaleza (Ceará) e Rio de Janeiro (Tabela 7).

As razões de chance de os óbitos de idosos serem registrados como causas mal definidas ou inespecíficas são apresentadas na Tabela 8. Para as mulheres, essa chance é aproximadamente $8 \%$ maior do que para os homens. Também foi observada uma relação direta entre a idade e a chance de ter causa mal definida. Idosos com idades avançadas (mais de 90 anos) são mais propensos a apresentar, no registro de óbito, causa mal definida do que aqueles com 60-69 anos, sendo quase quatro vezes maior a chance entre os idosos com 100 anos ou mais.

As características socioeconômicas dos idosos também se mostraram relacionadas com as causas mal definidas. Quanto à raça/cor, os resultados indicam que não ser branco aumenta a chance de ter causa mal definida, sendo que as maiores chances foram encontradas entre os indígenas (67\%). A escolaridade do idoso mostrouse inversamente associada com as causas mal definidas. Idosos sem escolaridade têm aproximadamente $135 \%$ a mais de chance de terem causas mal definidas quando comparados àqueles com 8 anos ou mais de estudo. Entre idosos com 1-3 anos e 4-7 anos de escolaridade, a chance foi $77 \%$ e $42 \%$ maior, respectivamente.

O tipo de atenção à morte dos idosos também se mostrou associado ao registro de causa mal definida na DO. Os que não tiveram assistência médica durante a doença que ocasionou o óbito, apresentaram aproximadamente 14 vezes mais de chance de ter causas mal definidas do que aqueles que tiveram assistência. Chances bem elevadas de ter causas mal definidas também foram observadas entre idosos cujo óbito ocorreu fora de estabelecimentos de saúde ou hospitais (tais como domicílio ou na rua).

A qualidade da informação sobre causas de morte mostrou-se associada com a completitude do preenchimento de algumas variáveis da DO. Idosos cujo "lugar de ocorrência do óbito" não foi preenchido têm aproximadamente 11 vezes mais chance de apresentar causa mal definida do que aqueles que faleceram em estabelecimentos de saúde. Óbito com informação ignorada na variável raça mostrou chance $60 \%$ maior de ter causa mal definida do que idosos de cor branca. A falta de informação sobre escolaridade também se mostrou associada: a chance de apresentar causa mal definida entre idosos cujo nível educacional não estava preenchido foi $91 \%$ maior do que para aqueles com oito ou mais anos de escolaridade.

Com relação às variáveis contextuais, observou-se que, quanto menor o porte do município maior é a chance de óbitos de idosos serem registrados com causa mal definida. As maiores chances foram encontradas nos municípios com população entre 20 e 50 mil habitantes. Residir em capitais reduz em $69 \%$ a chance de o óbito ser registrado com causa mal definida. Uma relação inversa também foi verificada entre o PIB per capita e a chance de registro de causa mal definida. 
Número e proporção de óbitos entre idosos por causas inespecíficas e participação das três principais causas inespecíficas, por Unidades da Federação (UF). Brasil, 2007.

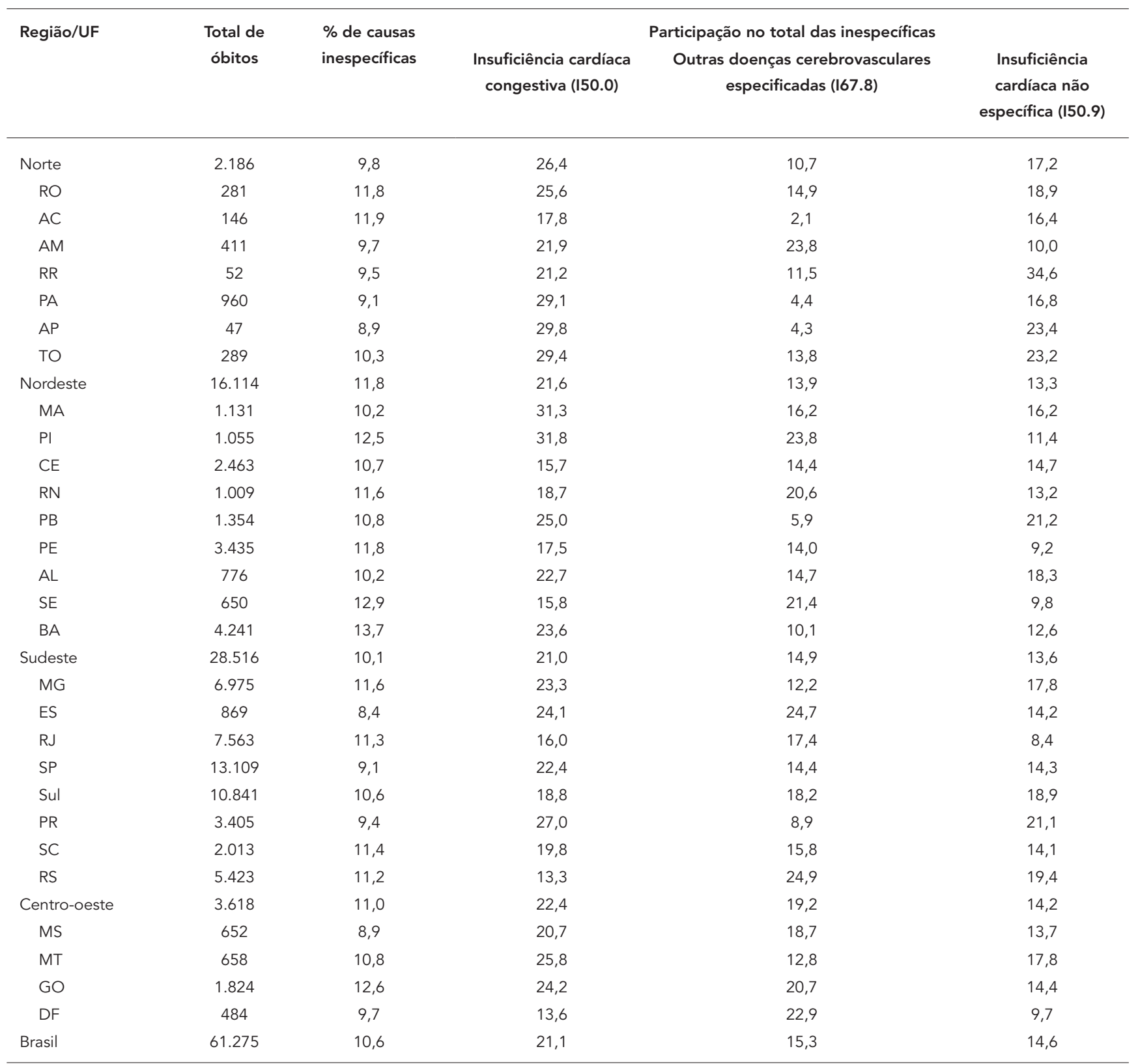

AC: Acre; AL: Alagoas; AM: Amazonas; AP: Amapá; BA: Bahia; CE: Ceará; DF: Distrito Federal; ES: Espírito Santo; GO: Goiás; MA: Maranhão; MG: Minas Gerais; MS: Mato Grosso do Sul; MT: Mato Grosso; PA: Pará; PB: Paraíba; PE: Pernambuco; PI: Piauí; PR: Paraná; RJ: Rio de Janeiro; RN: Rio Grande do Norte; RS: Rio Grande do Sul; RO: Rondônia; RR: Roraima; SC: Santa Catarina; SE: Sergipe; SP: São Paulo; TO: Tocantins.

Fonte: Sistema de Informações sobre Mortalidade (SIM), Ministério da Saúde.

As causas de morte inespecíficas mostraram associações semelhantes às mal definidas para as seguintes variáveis de nível individual: sexo, idade e anos de estudo. Em relação à raça/cor, apenas a categoria referente à cor preta mostrouse estatisticamente significativa. Idosos dessa cor apresentaram maiores chances de ter registrada em seu óbito uma causa inespecífica quando comparados aos brancos. No nível contextual, o tamanho do município e a variável indicativa de município capital também apresentaram relações semelhantes. Já os resultados das variáveis 
Tabela 5

Número e proporção de óbitos entre idosos por causas inespecíficas e participação das três principais causas inespecíficas, por capitais, Brasil, 2007.

\begin{tabular}{|c|c|c|c|c|c|}
\hline \multirow[t]{2}{*}{ Região/UF } & \multirow{2}{*}{$\begin{array}{l}\text { Total de } \\
\text { óbitos }\end{array}$} & \multirow{2}{*}{$\begin{array}{l}\% \text { de causas } \\
\text { inespecíficas }\end{array}$} & \multicolumn{3}{|c|}{ Participação no total das inespecíficas } \\
\hline & & & $\begin{array}{l}\text { Insuficiência cardíaca } \\
\text { congestiva (150.0) }\end{array}$ & $\begin{array}{c}\text { Outras doenças } \\
\text { cerebrovasculares } \\
\text { especificadas } \\
\text { (167.8) }\end{array}$ & $\begin{array}{l}\text { Insuficiência cardíaca não específica } \\
\qquad(150.9)\end{array}$ \\
\hline Norte & 787 & 8,7 & 20,2 & 13,7 & 14,2 \\
\hline $\mathrm{RO}$ & 83 & 11,5 & 20,5 & 15,7 & 26,5 \\
\hline$A C$ & 70 & 10,2 & 15,7 & 0,0 & 10,0 \\
\hline AM & 271 & 9,2 & 17,0 & 29,9 & 7,4 \\
\hline $\mathrm{RR}$ & 41 & 10,2 & 17,1 & 14,6 & 34,1 \\
\hline PA & 276 & 7,5 & 24,6 & 1,4 & 14,1 \\
\hline AP & 30 & 8,0 & 26,7 & 3,3 & 20,0 \\
\hline TO & 16 & 7,4 & 12,5 & 18,8 & 25,0 \\
\hline Nordeste & 4.096 & 12,6 & 12,4 & 20,3 & 6,9 \\
\hline MA & 212 & 9,0 & 21,2 & 37,7 & 4,2 \\
\hline $\mathrm{Pl}$ & 337 & 15,6 & 32,0 & 38,9 & 8,0 \\
\hline CE & 699 & 11,2 & 7,7 & 21,3 & 5,4 \\
\hline $\mathrm{RN}$ & 247 & 10,4 & 15,4 & 20,6 & 6,1 \\
\hline PB & 246 & 10,1 & 9,8 & 14,6 & 13,4 \\
\hline PE & 749 & 12,9 & 6,4 & 14,7 & 2,0 \\
\hline$A L$ & 309 & 11,9 & 14,2 & 23,9 & 20,1 \\
\hline SE & 219 & 14,1 & 9,6 & 26,9 & 4,6 \\
\hline BA & 1.078 & 15,2 & 11,7 & 13,2 & 6,8 \\
\hline Sudeste & 6.437 & 7,8 & 18,6 & 10,7 & 9,0 \\
\hline MG & 640 & 7,9 & 17,8 & 2,5 & 15,2 \\
\hline ES & 58 & 5,3 & 22,4 & 37,9 & 8,6 \\
\hline RJ & 3.427 & 11,0 & 12,8 & 17,9 & 6,1 \\
\hline SP & 2.312 & 5,5 & 27,4 & 1,6 & 11,5 \\
\hline Sul & 1.076 & 7,5 & 20,7 & 21,7 & 15,9 \\
\hline PR & 537 & 8,8 & 21,2 & 24 & 14,2 \\
\hline SC & 105 & 9,0 & 14,3 & 21,9 & 9,5 \\
\hline RS & 434 & 6,1 & 21,7 & 18,7 & 19,6 \\
\hline Centro-oeste & 1.239 & 9,9 & 16,5 & 25,3 & 11,2 \\
\hline MS & 215 & 8,9 & 12,1 & 29,3 & 10,2 \\
\hline MT & 132 & 10,1 & 21,2 & 25,8 & 16,7 \\
\hline GO & 408 & 10,8 & 20,6 & 26,0 & 11,8 \\
\hline DF & 484 & 9,7 & 13,6 & 22,9 & 9,7 \\
\hline Brasil & 13.635 & 9,0 & 16,8 & 15,9 & 9,4 \\
\hline
\end{tabular}

AC: Acre; AL: Alagoas; AM: Amazonas; AP: Amapá; BA: Bahia; CE: Ceará; DF: Distrito Federal; ES: Espírito Santo; GO: Goiás; MA: Maranhão; MG: Minas Gerais; MS: Mato Grosso do Sul; MT: Mato Grosso; PA: Pará; PB: Paraíba; PE: Pernambuco; PI: Piauí; PR: Paraná; RJ: Rio de Janeiro; RN: Rio Grande do Norte; RS: Rio Grande do Sul; RO: Rondônia; RR: Roraima; SC: Santa Catarina; SE: Sergipe; SP: São Paulo; TO: Tocantins; UF: Unidade da Federação.

Fonte: Sistema de Informações sobre Mortalidade (SIM), Ministério da Saúde.

de atenção à morte, assistência médica e local de ocorrência do óbito demonstraram associações em sentido oposto às observadas para as causas de morte mal definidas. Mortes sem assistência médica tiveram chance $19 \%$ menor de ter causa de morte inespecífica do que aquelas com assistência médica. Falecer no domicílio ou em outros locais diminui a chance de registro de inespecífica. 
Número e proporção de óbitos entre idosos por causas inespecíficas e participação das três principais causas inespecíficas, por municípios não-capitais. Brasil, 2007.

\begin{tabular}{|c|c|c|c|c|c|}
\hline \multirow[t]{2}{*}{ Região/UF } & \multirow{2}{*}{$\begin{array}{l}\text { Total de } \\
\text { óbitos }\end{array}$} & \multirow{2}{*}{$\begin{array}{l}\% \text { de causas } \\
\text { inespecíficas }\end{array}$} & \multicolumn{3}{|c|}{ Participação no total das inespecíficas } \\
\hline & & & $\begin{array}{l}\text { Insuficiência cardíaca } \\
\text { congestiva (I50.0) }\end{array}$ & $\begin{array}{c}\text { Outras doenças cerebrovasculares } \\
\text { especificadas (167.8) }\end{array}$ & $\begin{array}{c}\text { Insuficiência } \\
\text { cardíaca não } \\
\text { específica (I50.9) }\end{array}$ \\
\hline Norte & 1.399 & 10,5 & 29,9 & 8,9 & 18,8 \\
\hline $\mathrm{RO}$ & 198 & 12,0 & 27,8 & 14,6 & 15,7 \\
\hline$A C$ & 76 & 14,1 & 19,7 & 3,9 & 22,4 \\
\hline AM & 140 & 10,7 & 31,4 & 12,1 & 15,0 \\
\hline RR & 11 & 7,6 & 36,4 & 0,0 & 36,4 \\
\hline PA & 684 & 9,9 & 30,8 & 5,6 & 17,8 \\
\hline AP & 17 & 10,8 & 35,3 & 5,9 & 29,4 \\
\hline TO & 273 & 10,5 & 30,4 & 13,6 & 23,1 \\
\hline Nordeste & 12.018 & 11,6 & 24,8 & 11,7 & 15,5 \\
\hline MA & 919 & 10,5 & 33,6 & 11,2 & 18,9 \\
\hline $\mathrm{PI}$ & 718 & 11,4 & 31,8 & 16,7 & 13,0 \\
\hline $\mathrm{CE}$ & 1.764 & 10,5 & 18,9 & 11,7 & 18,4 \\
\hline $\mathrm{RN}$ & 762 & 12,0 & 19,8 & 20,6 & 15,5 \\
\hline PB & 1.108 & 11,0 & 28,4 & 4,0 & 22,9 \\
\hline PE & 2.686 & 11,5 & 20,6 & 13,8 & 11,2 \\
\hline $\mathrm{AL}$ & 467 & 9,3 & 28,3 & 8,6 & 17,1 \\
\hline SE & 431 & 12,4 & 19,0 & 18,6 & 12,5 \\
\hline BA & 3.163 & 13,2 & 27,7 & 9,0 & 14,6 \\
\hline Sudeste & 22.079 & 11,1 & 21,7 & 16,2 & 14,9 \\
\hline MG & 6.335 & 12,2 & 23,8 & 13,1 & 18,1 \\
\hline ES & 811 & 8,7 & 24,2 & 23,8 & 14,5 \\
\hline RJ & 4.136 & 11,5 & 18,7 & 16,9 & 10,2 \\
\hline $\mathrm{SP}$ & 10.797 & 10,6 & 21,4 & 17,1 & 14,9 \\
\hline Sul & 9.765 & 11,1 & 18,6 & 17,8 & 19,3 \\
\hline PR & 2.868 & 9,6 & 28,1 & 6,1 & 22,4 \\
\hline SC & 1.908 & 11,5 & 20,1 & 15,5 & 14,3 \\
\hline RS & 4.989 & 12,1 & 12,6 & 25,4 & 19,4 \\
\hline Centro-oeste & 2.379 & 11,6 & 25,6 & 16,0 & 15,8 \\
\hline MS & 437 & 8,9 & 24,9 & 13,5 & 15,3 \\
\hline MT & 526 & 11,0 & 27,0 & 9,5 & 18,1 \\
\hline GO & 1.416 & 13,2 & 25,2 & 19,1 & 15,1 \\
\hline DF & 0 & & & & \\
\hline Brasil & 47.640 & 11,2 & 22,3 & 15,2 & 16,1 \\
\hline
\end{tabular}

AC: Acre; AL: Alagoas; AM: Amazonas; AP: Amapá; BA: Bahia; CE: Ceará; DF: Distrito Federal; ES: Espírito Santo; GO: Goiás; MA: Maranhão; MG: Minas Gerais; MS: Mato Grosso do Sul; MT: Mato Grosso; PA: Pará; PB: Paraíba; PE: Pernambuco; PI: Piauí; PR: Paraná; RJ: Rio de Janeiro; RN: Rio Grande do Norte; RS: Rio Grande do Sul; RO: Rondônia; RR: Roraima; SC: Santa Catarina; SE: Sergipe; SP: São Paulo; TO: Tocantins; UF: Unidade da Federação.

Fonte: Sistema de Informações sobre Mortalidade (SIM), Ministério da Saúde.

Diferentemente do observado para as causas mal definidas, o não preenchimento das variáveis individuais, na DO, só mostrou relação estatisticamente significativa com a chance de ter causa de óbito inespecífica para as variáveis anos de estudo e assistência médica, mesmo assim com efeito bastante reduzido. A chance de ter causa inespecífica entre idosos com nível educacional não indicado foi $14 \%$ maior quando comparada a de idosos com 8 ou mais anos de escolaridade. 
Tabela 7

Classificação do indicador combinado da proporção de óbitos de idosos por causas mal definidas e inespecíficas, por Unidades da Federação (UF), capitais e municípios não-capitais. Brasil, 2007.

\begin{tabular}{|c|c|c|c|}
\hline Classificação & UF & Capitais & Municípios não-capitais \\
\hline Alta qualidade & TO, ES, MS, DF & $\begin{array}{c}A C, T O, M A, R N, P B, E S, S P, P R, S C, \\
\text { RS, MS, MT, DF }\end{array}$ & TO, CE, ES, MS \\
\hline Qualidade aceitável & $\mathrm{RR}, \mathrm{CE}, \mathrm{RN}, \mathrm{SP}, \mathrm{PR}, \mathrm{RS}, \mathrm{MT}$ & $R R, P I, P E, A L, S E, B A, M G, G O$ & $\mathrm{RN}, \mathrm{PR}, \mathrm{MT}$ \\
\hline Qualidade regular & $\mathrm{PI}, \mathrm{PE}, \mathrm{AL}$ & PA & $\mathrm{RR}, \mathrm{PI}, \mathrm{SP}, \mathrm{RS}$ \\
\hline Inadequado & $\begin{array}{c}R O, A C, A M, P A, A P, M A, P B, S E, B A \\
M G, R J, S C, G O\end{array}$ & $R O, A M, A P, C E, R J$ & $\begin{array}{c}R O, A C, A M, P A, A P, M A, P B, P E, A L, \\
\text { SE, BA, MG, RJ, SC, GO }\end{array}$ \\
\hline
\end{tabular}

AC: Acre; AL: Alagoas; AM: Amazonas; AP: Amapá; BA: Bahia; CE: Ceará; DF: Distrito Federal; ES: Espírito Santo; GO: Goiás; MA: Maranhão; MG: Minas Gerais; MS: Mato Grosso do Sul; MT: Mato Grosso; PA: Pará; PB: Paraíba; PE: Pernambuco; PI: Piauí; PR: Paraná; RJ: Rio de Janeiro; RN: Rio Grande do Norte; RS: Rio Grande do Sul; RO: Rondônia; RR: Roraima; SC: Santa Catarina; SE: Sergipe; SP: São Paulo; TO: Tocantins.

Fonte: Sistema de Informações sobre Mortalidade (SIM), Ministério da Saúde.

Tabela 8

Razões de chance de o idoso ter em seu registro de óbito causa mal definida ou causa inespecífica e seus respectivos intervalos de 95\% de confiança (IC95\%), segundo variáveis. Brasil, 2007.

\begin{tabular}{|c|c|c|c|c|}
\hline \multirow[t]{2}{*}{ Variáveis } & \multicolumn{2}{|c|}{ Causa mal definida } & \multicolumn{2}{|c|}{ Causa inespecífica } \\
\hline & $\begin{array}{c}\text { Razão de } \\
\text { chance }\end{array}$ & IC95\% & $\begin{array}{c}\text { Razão de } \\
\text { chance }\end{array}$ & IC95\% \\
\hline \multicolumn{5}{|l|}{ Individuais } \\
\hline \multicolumn{5}{|l|}{ Demográficas } \\
\hline \multicolumn{5}{|l|}{ Sexo } \\
\hline Homens & 1,00 & & 1,00 & \\
\hline Mulheres & 1,08 & $1,05-1,10$ & 1,14 & $1,12-1,16$ \\
\hline \multicolumn{5}{|l|}{ Idade (anos) } \\
\hline $60-69$ & 1,00 & & 1,00 & \\
\hline $70-79$ & 1,00 & $0,97-1,03$ & 1,17 & $1,14-1,20$ \\
\hline $80-89$ & 1,28 & $1,24-1,31$ & 1,44 & $1,41-1,47$ \\
\hline $90-99$ & 2,24 & $2,17-2,32$ & 1,79 & $1,74-1,85$ \\
\hline 100 e mais & 4,41 & $4,04-4,80$ & 1,92 & $1,74-2,11$ \\
\hline \multicolumn{5}{|l|}{ Socioeconômicas } \\
\hline \multicolumn{5}{|l|}{ Raça/cor } \\
\hline Branca & 1,00 & & 1,00 & \\
\hline Preta & 1,28 & $1,23-1,33$ & 1,09 & $1,06-1,13$ \\
\hline Amarela & 1,29 & $1,13-1,48$ & 0,96 & $0,85-1,08$ \\
\hline Parda & 1,24 & $1,21-1,28$ & 1,00 & $0,98-1,02$ \\
\hline Indígena & 1,67 & $1,34-2,09$ & 0,94 & $0,73-1,19$ \\
\hline Ignorada & 1,60 & $1,54-1,66$ & 1,01 & $0,98-1,05$ \\
\hline \multicolumn{5}{|c|}{ Anos de estudo } \\
\hline Nenhum & 2,35 & $2,24-2,47$ & 1,30 & $1,25-1,35$ \\
\hline $1-3$ & 1,77 & $1,69-1,86$ & 1,18 & $1,14-1,22$ \\
\hline $4-7$ & 1,42 & $1,35-1,50$ & 1,07 & $1,03-1,11$ \\
\hline 8 e mais & 1,00 & & 1,00 & \\
\hline Ignorado & 1,91 & $1,82-2,00$ & 1,14 & $1,10-1,18$ \\
\hline
\end{tabular}

(continua) 
Tabela 8 (continuação)

\begin{tabular}{|c|c|c|c|c|}
\hline \multirow[t]{2}{*}{ Variáveis } & \multicolumn{2}{|c|}{ Causa mal definida } & \multicolumn{2}{|c|}{ Causa inespecífica } \\
\hline & $\begin{array}{l}\text { Razão de } \\
\text { chance }\end{array}$ & IC95\% & $\begin{array}{l}\text { Razão de } \\
\text { chance }\end{array}$ & IC95\% \\
\hline \multicolumn{5}{|l|}{ Atenção à morte } \\
\hline \multicolumn{5}{|l|}{ Teve assistência médica } \\
\hline Sim & 1,00 & & 1,00 & \\
\hline Não & 14,11 & $13,60-14,65$ & 0,84 & $0,80-0,88$ \\
\hline Ignorado & 5,98 & $5,82-6,15$ & 1,03 & $1,01-1,05$ \\
\hline \multicolumn{5}{|l|}{ Local de ocorrência do óbito } \\
\hline Estabelecimento de saúde & 1,00 & & 1,00 & \\
\hline Domicílio & 8,42 & $8,23-8,62$ & 0,91 & $0,89-0,93$ \\
\hline Outros & 5,81 & $5,55-6,09$ & 0,81 & $0,77-0,86$ \\
\hline Ignorado & 11,19 & $9,68-12,94$ & 1,10 & $0,90-1,35$ \\
\hline \multicolumn{5}{|l|}{ Contextuais } \\
\hline \multicolumn{5}{|l|}{ Desenvolvimento } \\
\hline \multicolumn{5}{|l|}{ População do município } \\
\hline \multicolumn{5}{|l|}{ (habitantes) } \\
\hline$<20.000$ & 3,01 & $2,03-4,47$ & 1,23 & $1,12-1,36$ \\
\hline $20.000-50.000$ & 3,56 & $2,39-5,32$ & 1,20 & $1,08-1,33$ \\
\hline $50.000-100.000$ & 2,99 & $1,97-4,54$ & 1,21 & $1,09-1,35$ \\
\hline $100.000-500.000$ & 1,84 & $1,21-2,81$ & 1,09 & $0,98-1,22$ \\
\hline$>500.000$ & 1,00 & & 1,00 & \\
\hline \multicolumn{5}{|l|}{ Capital } \\
\hline Sim & 0,31 & $0,19-0,50$ & 0,85 & $0,75-0,96$ \\
\hline Não & 1,00 & & 1,00 & \\
\hline \multicolumn{5}{|l|}{ Pobreza } \\
\hline PIB per capita (por $\mathrm{R} \$ 1.000)$ & 0,98 & $0,98-0,98$ & 1,00 & $1,00-1,00$ \\
\hline
\end{tabular}

Fonte: Sistema de Informações sobre Mortalidade (SIM), Ministério da Saúde.

Já em relação à assistência médica, esse aumento foi de apenas $3 \%$.

\section{Discussão}

A informação sobre a causa básica de morte é fundamental para a estimativa de indicadores 14,22 utilizados no processo de avaliação e monitoramento das condições de saúde, assim como para o planejamento de ações adequadas à saúde pública 1 . No Brasil, dos 18 indicadores de mortalidade selecionados pela Rede Interagencial de Informações para a Saúde (RIPSA) 12, têm como fonte de dados a causa básica de morte.

A maioria dos trabalhos sobre a qualidade da informação da causa de morte focaliza-se nas crianças, especialmente nos menores de um ano $15,16,23,24$, e em mulheres em idade reprodutiva $22,25,26,27$. Poucas são as análises voltadas para a população idosa 7,13 , sendo a maior parte baseada apenas nas causas mal definidas. Em recente artigo, Lima-Costa et al. 11 analisam, além das causas mal definidas, as causas inespecíficas, porém, a população considerada restringe-se a uma coorte de idosos de Bambuí, município de Minas Gerais.

No presente trabalho, analisaram-se tanto as causas de óbitos mal definidas como as inespecíficas registradas entre os idosos, por sexo e idade, não só para o total do Brasil, mas também para as macrorregiões e UF, desagregadas em municípios capitais e não-capitais. Também foram estudadas as principais causas de morte que compõem o grupo de causas mal definidas e inespecíficas, salientando-se as desigualdades socioeconômicas e demográficas no que diz respeito à qualidade da informação sobre a causa básica de morte. Por fim, por meio de modelos multinomiais hierárquicos, avaliou-se a associação entre a qualidade da informação e um conjunto de variáveis individuais e contextuais.

No SIM foram registrados, para o Brasil, 628.488 óbitos de idosos, em 2007, dos quais $113.779(18,1 \%)$ não tiveram a causa de morte adequadamente definida, seja porque foi classi- 
ficada no capítulo das mal definidas $(8,4 \%)$, seja porque não estava suficientemente especificada (10,6\%). Essa proporção encontra-se num patamar bem acima do valor mediano de $12 \%$ encontrado por Mathers et al. ${ }^{3}$ para a população mundial. Entretanto, a comparação deve considerar que a pesquisa desses autores refere-se à população geral e não apenas ao grupo etário de idosos.

Os resultados mostraram que a qualidade da informação da causa básica de morte piora na medida em que a idade avança. A proporção de causas mal definidas foi ligeiramente mais elevada entre os homens, enquanto a proporção de inespecíficas foi maior entre as mulheres.

A proporção de causas inespecíficas é mais elevada do que a das mal definidas, entre idosos. Isso poderia ser resultado das "condições clínicas”, pois, com a velhice, torna-se mais difícil estabelecer um diagnóstico preciso da causa de óbito devido à coexistência de diversas doenças crônicas 28,29,30. Outro fator importante diz respeito às condições sociológicas do envelhecimento. O preconceito sobre a velhice e o "desconforto peculiar sentido pelos vivos na presença dos moribundos" 31 (p. 31), assim como a ideia da inexorável morte, poderiam resultar num interesse menor dos profissionais de saúde em descrever precisamente as causas de morte do idoso. Tais hipóteses merecem ser aprofundadas por meio de estudos qualitativos.

A qualidade da informação da causa de morte apresenta fortes diferenciais geográficos e socioeconômicos 32 , com pior qualidade nas áreas mais pobres 33,34 , no que diz respeito ao indicador combinado de causas mal definidas e inespecíficas. No presente trabalho observou-se que a qualidade da informação, na maioria das capitais brasileiras, é alta e aceitável, enquanto que em grande parte dos municípios não-capitais é regular ou inadequada. A melhor qualidade das informações sobre causas de óbitos foi verificada em Mato Grosso do Sul, com aproximadamente 90\% de causas bem definidas; entretanto, devese considerar que, mesmo nessa UF, esforços devem ser realizados para melhorar a informação das causas de mortalidade dos idosos.

Os diferenciais geográficos da qualidade da informação de causa de morte dos idosos são mais acentuados para o indicador de proporção de causas mal definidas do que para o das inespecíficas. As áreas do país consideradas mais desenvolvidas, especialmente as capitais das Regiões Sudeste, Sul e Centro-oeste, apresentaram as menores proporções de causas mal definidas, em consonância com estudos voltados para registros de óbitos em menores de um ano 5 , o que poderia ser explicado pelo maior acesso aos serviços de saúde ${ }^{32}$. Embora a proporção de causas mal definidas esteja em patamares baixos nas áreas mais desenvolvidas, as causas inespecíficas ainda registram valores elevados, prejudicando a qualidade da informação de causa de morte 34 .

Ao analisarem-se de forma desagregada as causas mal definidas, verificou-se que a "morte sem assistência" foi a mais frequente, especialmente nas Regiões Norte e Nordeste e municípios não-capitais. Cabe destacar que em Porto Velho, Palmas, João Pessoa e Recife não é utilizada a categoria "morte sem assistência”, o que poderia ser resultado de critérios normativos locais da classificação de óbitos com causas mal definidas. Na CID-10, a "morte sem assistência” é definida nos casos em que o indivíduo é "encontrado(a) morto(a)" ou "morte em circunstâncias nas quais o corpo do(a) falecido(a) foi encontrado e não se pode descobrir a causa" 19 (p. 890), refletindo a falta de acesso aos serviços de saúde e a qualidade do atendimento médico recebido nessas localidades 7,35. Os resultados dos modelos reforçam essa hipótese ao mostrarem que idosos residentes em capitais ou que faleceram em estabelecimentos de saúde apresentaram chances menores de ter causa de morte mal definida, quando comparados àqueles que morreram em outros locais. Além disso, quanto menor a população do município de residência maior é a chance da causa básica de morte ser mal definida.

Entre as três principais causas inespecíficas, predominam as insuficiências cardíacas (congestiva ou não específica), além do grupo de outras doenças cerebrovasculares especificadas. No Brasil, essas causas correspondem a $50 \%$ de todas as causas de morte inespecíficas entre idosos elencadas neste estudo. Parte das doenças cardiovasculares foi classificada entre as causas mal definidas e parte entre as inespecíficas. A soma dos óbitos devido à parada respiratória (mal definidas) e às insuficiências cardíacas (inespecíficas) corresponde a aproximadamente $30 \%$, sendo maior nas áreas mais pobres e nos municípios menores.

Neste trabalho propôs-se uma lista de causas de mortalidade inespecíficas para idosos que deve ser atualizada e levada a consenso, considerando-se divergências quanto à inclusão de algumas causas de mortalidade, como, por exemplo, insuficiência cardíaca. Alguns autores defendem que essa causa é uma condição crônica, sendo o evento final geralmente um edema pulmonar ou choque cardiogênico 36 e, portanto, deveria ser classificada como inespecífica. Outros argumentam que a insuficiência cardíaca como causa básica de morte é uma informação extremamente útil sob o ponto de vista epidemiológico, não devendo ser incluída no rol das inespecíficas 37,38. 
As características socioeconômicas dos idosos mostraram-se associadas à qualidade da informação sobre as causas de morte, com maior intensidade para as mal definidas do que para as inespecíficas. Municípios menores e mais pobres apresentaram pior qualidade de informação, devendo, desta forma, receber mais atenção na elaboração de programas e políticas.

O problema da qualidade da informação sobre a causa básica de morte não se mostrou independente da qualidade do preenchimento das demais variáveis da DO 25,39. A associação entre as causas mal definidas e o não preenchimento das características socioeconômicas e, principalmente, de atenção à saúde é bastante forte. Já entre as inespecíficas, essa associação ocorreu apenas em relação às variáveis anos de estudo e assistência médica, e com intensidade bem menor. Argumenta-se que a pouca importância dada ao ensino sobre o preenchimento dos formulários referentes aos registros vitais e a baixa valorização da DO como documento de grande relevância epidemiológica, contribuem sensivelmente para a deficiência na qualidade da informação 39,40,41.

\section{Resumo}

Identificaram-se diferenciais geográficos e socioeconômicos do padrão de qualidade da informação sobre a causa básica de morte dos idosos no Brasil. Utilizaram-se três indicadores de qualidade: proporção de causas mal definidas; proporção de causas inespecíficas; e a combinação de ambas. Analisaram-se as principais causas mal definidas e inespecíficas e a associação destes indicadores com características individuais constantes na Declaração de Óbito (DO) e contextuais dos municípios. A proporção de causas inespecíficas é maior do que a de mal definidas e ambas aumentam com a idade. Escolaridade, cor/raça, porte do municí-
As causas inespecíficas não têm sido focalizadas nas políticas nacionais, fazendo-se necessária a implantação de ações que visem à sua redução 11. Recomenda-se que programas voltados para a melhoria da qualidade da informação objetivem a redução do Registro de Óbitos não somente com causas mal definidas 4 , mas também com causas inespecíficas.

Há alguns aspectos metodológicos que merecem ser abordados. A classificação dos municípios em capitais e não-capitais pode constituir uma limitação para o estudo, pois estas duas categorias não captam toda a heterogeneidade existente, principalmente entre os municípios nãocapitais. Dessa forma, novos recortes geográficos deveriam ser avaliados. Outra limitação refere-se ao reduzido número de variáveis demográficas e socioeconômicas disponíveis na base do SIM, limitando de certa forma as análises sobre desigualdades sociais. Resultados não apresentados do modelo hierárquico revelam que existe uma grande variabilidade nos dados não explicada pelas variáveis selecionadas, apontando para a necessidade de novos estudos de cunho não só quantitativo, mas também qualitativo. pio e PIB per capita mostraram-se associados com a qualidade da informação, mas de forma mais intensa com as causas mal definidas do que com as inespecíficas. Ter recebido assistência médica diminui a chance de o óbito do idoso ser registrado como causas mal definidas e aumenta a de inespecíficas. Programas voltados para a melhoria da qualidade da informação da DO devem focar não apenas as causas de morte mal definidas entre os idosos, mas também as inespecíficas.

Declaração de Óbito; Causa Básica de Morte; Idoso 


\section{Colaboradores}

S. Kanso participou de todas as etapas: revisão da literatura, definição da metodologia utilizada, processamento dos dados, análises estatísticas, interpretação, discussão dos resultados e redação final do artigo. D. E. Romero e I. C. Leite contribuíram na revisão da literatura, definição da metodologia utilizada, análises estatísticas, interpretação, discussão dos resultados e redação final do artigo. E. N. Moraes contribuiu na definição da metodologia utilizada.

\section{Referências}

1. United Nations. Principios y recomendaciones para un sistema de estadísticas vitales. New York: United Nations; 1974. (Informes Estadísticos, serie M, 19).

2. Sibai AM. Mortality certification and cause-ofdeath reporting in developing countries. Bull World Health Organ 2004; 82:83

3. Mathers CD, Fat DM, Inoue M, Rao C, Lopez AD. Counting the dead and what they did from: an assessment of the global status of cause of death data Bull World Health Organ 2005; 83:171-7.

4. Ministério da Saúde. Manual para investigação do óbito com causa mal definida. Brasília: Ministério da Saúde; 2009. (Série A. Normas e Manuais Técnicos).

5. Szwarcwald CL, Leal MC, Andrade CLT, Souza Jr. PRB. Estimação da mortalidade infantil no Brasil: o que dizem as informações sobre óbitos e nascimentos do Ministério da Saúde? Cad Saúde Pública $2002 ; 18: 1725-36$.

6. Vasconcelos AMN. Qualidade das estatísticas de óbitos no Brasil: uma classificação das Unidades da Federação. In: Anais do XIV Encontro de Estudos Populacionais. Caxambu: Associação Brasileira de Estudos Populacionais; 2000. http://www. abep.nepo.unicamp.br/docs/anais/pdf/2000/ Todos/saut7_1.pdf (acessado em 28/Jan/2011).

7. Mello-Jorge MHP, Laurenti R, Lima-Costa MF, Gotlieb S, Chiavegatto Filho ADP. A mortalidade de idosos no Brasil: a questão das causas mal definidas. Epidemiol Serv Saúde 2008; 17:271-81.
8. Organização Mundial da Saúde. Classificação estatística internacional de doenças e problemas relacionados à saúde. 10a Revisão. v. 1. São Paulo: Centro Colaborador da OMS para a Classificação de Doenças em Português/Edusp; 1993.

9. Silvi J. On the estimation of mortality rates in countries of the Americas. Epidemiol Bull 2003; 24:1-5.

10. Costa MR, Marcopito LF. Mortalidade por causas mal definidas, Brasil, 1979-2002, e um modelo preditivo para idade. Cad Saúde Pública 2008; 24:1001-12.

11. Lima-Costa MF, Matos DL, Laurenti R, Mello-Jorge MHP, Cesar CC. Time trends and predictors of mortality from ill-defi ned causes in old age: 9 year folllow-up of the Bambuí cohort study (Brazil). Cad Saúde Pública 2010; 26:514-22.

12. Organização Pan-Americana da Saúde. IDB 2008 Brasil. Indicadores e dados básicos para a saúde. Rio de Janeiro: Ministério da Saúde/Organização Pan Americana da Saúde; 2009.

13. Abreu DMX, Sakurai E, Campos LN. A evolução da mortalidade por causas mal definidas na população idosa em quatro capitais brasileiras, 19962007. Rev Bras Estud Popul 2010; 27:75-88.

14. Murray CJL, Lopez AD. The Global Burden of Disease. Geneva: World Health Organization/Harvard School of Public Health/World Bank; 1996.

15. Santa-Helena, ET, Rosa, MB. Avaliação da qualidade das informações relativas aos óbitos em menores de um ano em Blumenau, 1998. Rev Bras Saúde Matern Infant 2003; 3:75-83. 
16. Soares JAS, Horta FMB, Caldeira AP. Avaliação da qualidade das informações em declarações de óbitos infantis. Rev Bras Saúde Matern Infant 2007; 7:289-95.

17. Rede Interagencial de Informações para a Saúde. Comentários sobre os Indicadores de Mortalidade. http://tabnet.datasus.gov.br/tabdata/ livroidb/Com2007/Com_C05.pdf (acessado em 20/ Mar/2011).

18. Organização Pan-Americana da Saúde. Brazil health system profile. Brasília: Organização PanAmericana da Saúde; 2005.

19. World Health Organization. National burden of disease Studies: a practical guide. Global Program on Evidence for Health Policy. Geneva: World Health Organization; 2001.

20. Hox JJ. Multilevel analysis: techniques and applications. Mahwah: Erlbaum 2002.

21. Goldstein H. Multilevel statistical models. 3rd Ed. London: Arnold; 2003.

22. Laurenti R, Mello-Jorge MHP, Gotlieb SLD. A confiabilidade dos dados de mortalidade e morbidade por doenças crônicas não-transmissíveis. Ciênc Saúde Coletiva 2009; 9:909-20.

23. Vidal AS, Frias PG, Barreto FMP, Vanderlei LCM, Felisberto E. Óbitos infantis evitáveis em hospital de referência estadual do Nordeste brasileiro. Rev Bras Saúde Matern Infant 2003; 3:281-9.

24. Gomes JO, Santo AH. Mortalidade infantil em município da região Centro-oeste Paulista, Brasil, 1990 a 1992. Rev Saúde Pública 1997; 31:330-41.

25. Chiavegatto Filho ADP, Laurenti R, Gotlieb SLD, Mello-Jorge MHP. Desnutrição como causa básica ou associada de morte: análise da qualidade de informação em mulheres em idade fértil. Rev Bras Epidemiol 2007: 10:30-8.

26. Chiagevatto Filho ADP, Laurenti R, Gotlieb SLD, Mello-Jorge MHP. Mortalidade por doença hipertensiva em mulheres de 20 a 49 anos no Município de São Paulo, SP, Brasil. Rev Bras Epidemiol 2004; 7:252-8.

27. Siqueira AA, Rio EM, Tanaka AC, Schor N, Alvarenga AT, Almeida LC. Mortalidade feminina na região Sul do Município de São Paulo: qualidade da certificação médica dos óbitos. Rev Saúde Pública 1999; 33:499-504.

28. Battle RM, Pathak D, Hule CG, Key CR, Vanatta PR, Hill RB, et al. Factors influencing discrepancies between premortem and postmortem diagnoses. JAMA 1987; 258:339-44.

29. Lloyd-Jones DM, Martin DO, Larson MG, Levy D. Accuracy of death certificates for coding coronary heart disease as the cause of death. Ann Intern Med 1998; 129:1020-6.
30. Vasconcelos AMN. Estatísticas de mortalidade por causas: uma avaliação da qualidade da informação In: Anais do X Encontro Nacional de Estudos Populacionais. Belo Horizonte: Associação Brasileira de Estudos Populacionais; 1996. p. 151-65.

31. Elias N. A solidão dos moribundos - seguido de "Envelhecer e morrer". Rio de Janeiro: Jorge Zahar Editor; 2001

32. Travassos C, Viacava F, Fernandes C, Almeida CM. Desigualdades geográficas e sociais na utilização de serviços de saúde no Brasil. Ciênc Saúde Coletiva 2000; 5:133-49.

33. Paes NA. Qualidade das estatísticas de óbitos por causas desconhecidas dos Estados brasileiros. Rev Saúde Pública 2007; 41:436-45.

34. França E, Abreu DX, Rao C, Lopes AD. Evaluation of cause-of-death statistics for Brazil, 2002-2004. Int J Epidemiol 2008; 37:891-901.

35. Santo AH. Causas mal definidas de morte e óbitos sem assistência. Rev Assoc Med Bras (1992) 2008; 54:23-8.

36. Laurenti R, Mello-Jorge MHP, Gotlieb SLD. Mortalidade segundo causas: considerações sobre a fidedignidade dos dados. Rev Panam Salud Pública 2008; 23:349-56.

37. Hunt SA, Abraham WT, Chin MH, Feldman AM, Francis GS, Ganiats TG, et al. 2009 focused update incorporated into the ACC/AHA 2005 Guidelines for the Diagnosis and Management of Heart Failure in Adults: a report of the American College of Cardiology Foundation/American Heart Association Task Force on Practice Guidelines: developed in collaboration with the International Society for Heart and Lung Transplantation. Circulation 2009; 119:e391-479.

38. Kalogeropoulos A, Georgiopoulou V, Kritchevsky SB, Psaty BM, Smith NL, Newman AB, et al. Epidemiology of incident heart failure in a contemporary elderly cohort: the health, aging, and body composition study. Arch Intern Med 2009; 169: 708-15.

39. Stuque CO, Cordeiro JA, Cury PM. Avaliação dos erros ou falhas de preenchimento dos atestados de óbito feitos pelos clínicos e pelos patologistas. J Bras Patol Med Lab 2003; 39:361-4.

40. Fonseca LAM, Laurenti R. A qualidade da certificação médica da causa de morte em São Paulo. Rev Saúde Pública 1974; 9:21-9.

41. Laurenti R. Fatores de erros na mensuração da mortalidade infantil. Rev Saúde Pública 1975; 9:529-37.

Recebido em 17/Set/2010

Versão final reapresentada em 11/Abr/2011

Aprovado em 18/Mai/2011 